\title{
EQUITABLE DISTRIBUTION OF COVID-19 VACCINES
}

\author{
ASM ALAMGIR
}

The COVID-19 pandemic is one of the most disruptive medical, social, political and economic crises of the modern era. In today's interconnected world, the pandemic shows how quickly infectious disease outbreaks can spread across continents. Since the initial outbreak, the introduction of several vaccines has brought hope to a virus-weary world. In spite of the remarkable results of approved vaccines, many lower-middle countries are yet to receive a single vaccine shot. While many wealthy nations have ramped up vaccination efforts and cautiously opened their borders, many in the developed world are still waiting to be inoculated.

The past twenty months have exposed the world to an extraordinary challenge with unprecedented setbacks and devastating social and economic repercussions that have forced usto learn [in order] to live'. Fortunately, we have done much learning and the pace at which the world has gained scientific knowledge about the SARS-Cov-2 virus has enabled the development of effective vaccines, which offer a glimpse of light at the end of the tunnel.

And yet, this scientific feat has not been matched by a commensurate effort in international cooperation and solidarity to ensure equitable global distribution of these live-saving vaccines. Instead, vaccine distribution has been grossly inequitable, resulting in terrible consequences for the enjoyment of human rights, such as the rights to health and to life, particularly in low-income countries. Of the 1.8 billion vaccines administered around the world, just $0.4 \%$ have been in low-income countries, which explains the recent surge of infections in the world's poorest countries and underlines once more the need, as repeatedly emphasised by WHO Director-General, TedrosAdhanomGhebreyesus, to ensure equitable vaccine access and distribution. Vaccine nationalism must give way to vaccine multilateralism that privileges increased global vaccine production, removal of export barriers, up scaling of COVAX, and the sharing of excess vaccines.

To beat Covid19, there's a simple lesson - "no one is safe until everyone is safe". Uncontrolled transmission means rapid viral replication and mutation, which in turn means huge opportunities for the emergence of new, more transmissible variants that could escape natural or vaccine-induced immunity.

Covid-19 vaccines shows a way-out of this disastrous pandemic, but bold and innovativepolicies that ensure faster and equitable distribution are also important.á Indeed, the $55-70 \%$ vaccine coverage among adults in the USA, UK, and EU stands in sharp contrast to the other world regions-only $4 \%$ of the population of Africa has been vaccinated, and more than 50 countries worldwide failed to meet the WHO target of fully vaccinating at least $10 \%$ of their populations by the end of September, 2021. 80\% of the roughly 5.5 billion doses of vaccine administered so far have gone to high-income and upper-middle-income countries. Of more than a billion doses of vaccine that high-income countries have pledged to donate, less than $20 \%$ have been delivered so far. ${ }^{2}$

If lifesaving COVID-19 vaccines are not made accessible and affordable for all right now, low-income countries would need to increase health care spending by $30-60 \%$ to vaccinate $70 \%$ of their populations - a daunting task for the world's poorest countries. Even if achieved, this would have major ramifications for other pressing health and development challenges. In contrast, high-income countries would have to increase their spending by just $0.8 \%$ to achieve the same level of protection.

All approved vaccines seem to show reasonable efficacy against symptomatic and severe disease after infection with the Delta variant in datasets from the United Kingdom and Canada.

Principal Scientific Officer, Institute of Epidemiology, Disease Control and Research (IEDCR), Dhaka, Bangladesh. DOI: https://doi.org/10.3329/bjm.v33i1.56781

Bangladesh J Medicine 2022; 33: 1-2

Copyright: (C) 2021 Association of Physicians of Bangladesh 
As a minority of countries consider offering booster doses of COVID-19 vaccines to their citizens, the majority are yet to vaccinate their entire populations with a single dose. ${ }^{3}$ As new variants emerge, breakthrough cases emerge, and a small number of these patients will develop complications, but no data exist that link boosters to enhanced real-life protection in the general population. Other than rare immunosuppressed patients, such as organtransplant recipients, the vast majority of the population, including the elderly and those with medical conditions, derive adequate protection from the existing vaccine schedule. Vaccinating the unvaccinated can save many more lives than would boosting people who already have a fairly high level of protection.

Vaccinating the world is not only a moral obligation to protect our neighbors, it also serves our selfinterest by protecting our security, health, and economy. These goals will not be accomplished by making the world wait for wealthy countries to be vaccinated first. By investing in multilateral partnerships with a sense of shared commitment and employing a global allocation strategy that increases supply and manufacturing, we can meet the urgent challenge of Covid-19, while creating sustainable infrastructures and health systems for the future. Getting the world vaccinated may well be the critical test of our time.

From an equity perspective, there is a real risk of worsening the existing global inequity in vaccinations.
There remain 3.5 billion people on this earth, nearly half of the world's population, who have not received even a first dose of vaccine. Only $4 \%$ of the African population has received a single dose.

The high risk people in developed countries have already been prioritised; vulnerable people in under developed countries cannot wait until 2022 for their turn, and this wait is in the best interest of no one.

No one should have that opportunity taken away from them just because their country does not have the necessary resources to provide it to their citizens.

It is time to realize that this pandemic is far from over and it won't be the last pandemic.

\section{References:}

1. Perspective: From Vaccine Nationalism to Vaccine Equity - Finding a Path Forward. Ingrid T. Katz, M.D., M.H.S., Rebecca Weintraub, M.D., Linda-Gail Bekker, M.D., Ph.D., and Allan M. Brandt, Ph.D. April 8, 2021, N Engl J Med 2021; 384:12811283DOI: $10.1056 /$ NEJM p2103614

2. Editorial: COVID-19: is the rush to boost backed by science? The Lancet Rheumatology;VOLUME 3, ISSUE 11, E737, NOVEMBER 01, 2021. November, 2021 DOI:https: / / doi.org/10.1016/S26659913(21)00330-1

3. Editorial: COVID-19 vaccine equity and booster dosesThe Lancet Infectious Diseases. , 12, 2021 DOI:https://doi.org/10.1016/S1473-3099 (21)00486-2. 